\title{
DEFORMATION OF A SPHERICAL MOLECULE
}

\author{
M. Apostol \\ Department of Theoretical Physics, Institute of Atomic Physics \\ Magurele-Bucharest MG-6, P.O. Box MG-35, Romania
}

(Reccived December 6, 1994)

\begin{abstract}
The deformation suffered by a hollow, spherical molecule as an effect of rotations is studied within a continuum model which might be relevant for the fullerene molecule. The spherical elasticity is established to the lowest order in deformations and the natural perturbation parameter required by the stability of the object is identified as the square ratio of the rotation to the radial vibration pulsations. It is also shown that the main effect of the centrifugal coupling between rotations and radial vibrations is a static deformation whose energy is beyond the harmonic approximation.
\end{abstract}

PACS numbers: 46.30.-i, 33.20.-t, 61.46.+w

\section{Introduction}

The advent of the $\mathrm{C}_{60}$ fullerene $[1,2]$ drew the attention to a new microscopic object: a hollow, spherical molecule composed of a large number of atoms. Electron structure [3-5], molecular polarizability $[6,7]$ and ion dynamics $[8,9]$ of such an object have extensively been studied, many of the molecular vibrations having been identified by various spectroscopic techniques [10-12], most notably in Raman and infrared spectra [13]. Comparatively litile attention was given to a certain class of low-energy molecular states, which may show themselves in the thermal properties [14], consisting of rotations and long-wavelength vibrations that do not involve an electric moment. These low-energy molecular states are addressed in the present paper.

As a consequence of the hollow spherical shape and the large number of atoms this molecule might easily be distorted by rotation and it might also easily vibrate, especially along the radius. Within a continuum model of a homogeneous, spherical shell of atoms of zero thickness the elastic energy is derived to the lowest order in deformations and the rotation spectrum of the deformed molecule is obtained. The stability of the rotating molecule requires a natural perturbation parameter which is the square ratio of the rotation to the radial vibration pulsations. The usual Coriolis coupling between rotations and vibrations is absent in the case of the 
radial vibrations, but the centrifugal coupling contains both linear and quadratic terms in displacements. By means of the above-mentioned perturbation parameter their contributions are easily classified; leading to the conclusion that the main effect of this coupling is a static deformation of the molecule which contributes a higher-order correction to the harmonic-approximation energy.

\section{Spherical elasticity}

We consider a spherical, homogeneous shell of atoms of radius $R$ and zero thickness whose points are located at

$$
\boldsymbol{R}=R(\sin \theta \cos \varphi, \sin \theta \sin \varphi, \cos \theta),
$$

where $0 \leq \theta<\pi$ and $0 \leq \varphi<2 \pi$. The natural representation of a displacement field on the sphere is given in spherical coordinates by

$$
\left\{\begin{array}{l}
\tilde{\theta}=\theta+u_{1}(\theta, \varphi), \\
\tilde{\varphi}=\varphi+u_{2}(\theta, \varphi), \\
\tilde{R}=R\left[1+u_{3}(\theta, \varphi)\right],
\end{array}\right.
$$

where $\left|u_{i}\right| \ll 1, i=1,2,3$. Generally, a displacement field includes translations, rotations and deformations. In order to establish the elastic (deformation) energy of a continuum [15] one needs the infinitesimal absolute length, i.e. the length which is invariant under translations and rotations: the changes underwent by this length during a displacement provide a measure for the elastic energy. The Cartesian length on the sphere $R^{2}\left(\mathrm{~d} \theta^{2}+\sin ^{2} \theta \mathrm{d} \varphi^{2}\right)$ is not adequate for this purpose since it is not invariant under $\theta$-rotations (translations) ${ }^{*}$. Instead, the absolute length on the sphere is provided by

$$
\mathrm{d} A^{2}=R^{2}\left(\mathrm{~d} \theta^{2}+\mathrm{d} \varphi^{2}\right)=\mathrm{d} \xi_{i} \mathrm{~d} \xi_{i},
$$

with the obvious notations $\mathrm{d} \xi_{1}=R \mathrm{~d} \theta, \mathrm{d} \xi_{2}=R \mathrm{~d} \varphi$ and the dummy summation over the repeating subscript $i=1,2$. Under the action of the displacement field (1) this length changes as

$$
\begin{aligned}
\mathrm{d} \tilde{A}^{2} & =R^{2}\left\{\left[\left(1+u_{11}\right) \mathrm{d} \theta+u_{12} \mathrm{~d} \varphi\right]^{2}+\left[u_{21} \mathrm{~d} \theta+\left(1+u_{22}\right) \mathrm{d} \varphi\right]^{2}\right\} \\
& \approx \mathrm{d} A^{2}+2 u_{i j} \mathrm{~d} \xi_{i} d \xi_{j}
\end{aligned}
$$

where

$$
u_{i j}=\frac{R}{2}\left(\frac{\partial u_{i}}{\partial \xi_{j}}+\frac{\partial u_{j}}{\partial \xi_{i}}\right)
$$

is the deformation (strain) tensor (with respect to the rotations about the $\boldsymbol{R}$-axis) and only the first-order contributions in $u_{i j}$ have been retained. The deformation tensor (5) is invariant under $\theta$ - and $\varphi$-rotations (translations), comprises dilations and compressions $\left(u_{11}, u_{22}\right)$ as well as shear deformations $\left(u_{12}\right)$ and does not contain the $\boldsymbol{R}$-rotations (described by its antisymmetric counterpart).

*The Cartesian length is relevant for the usual elasticity which applies to a three-dimensional continuum, as for example a spherical shell of finite thickness, much larger than the displacements. 
When the radial displacement is included, the infinitesimal length is given by

$$
\begin{aligned}
\mathrm{d} \tilde{l}^{2} & =\left(1+u_{3}\right)^{2} \mathrm{~d} \tilde{A}^{2}+R^{2} \mathrm{~d} u_{3}^{2} \approx \mathrm{d} \tilde{A}^{2}+2 u_{3} \mathrm{~d} A^{2} \\
& \approx \mathrm{d} \dot{A}^{2}+2 u_{3} \mathrm{~d} \xi_{i} \mathrm{~d} \xi_{i}+2 u_{i j} \mathrm{~d} \xi_{i} \mathrm{~d} \xi_{j},
\end{aligned}
$$

whence one can see that the elastic energy depends on $u_{3}$ and $u_{i j}$ to the lowest order of approximation. The local isotropy of the shell requires the elastic energy be invariant under the rotations about the $\boldsymbol{R}$-axis. There are only four such quadratic invariants (harmonic approximation),

$$
u_{3}^{2}, u_{3} u_{i i}, u_{i i}^{2}, u_{i j}^{2}
$$

whence one can write down the elastic energy of the homogeneous shell as

$$
\begin{aligned}
E_{\text {el }} & =\sigma \int \mathrm{d} S u_{3}^{2}+\lambda \int \mathrm{d} S u_{3}\left(\frac{\partial u_{1}}{\partial \theta}+\frac{\partial u_{2}}{\partial \varphi}\right)+\mu \int \mathrm{d} S\left(\frac{\partial u_{1}}{\partial \theta}+\frac{\partial u_{2}}{\partial \varphi}\right)^{2} \\
& +\tau \int \mathrm{d} S\left[\left(\frac{\partial u_{1}}{\partial \theta}\right)^{2}+\left(\frac{\partial u_{2}}{\partial \varphi}\right)^{2}+\frac{1}{2}\left(\frac{\partial u_{1}}{\partial \varphi}+\frac{\partial u_{2}}{\partial \theta}\right)^{2}\right]
\end{aligned}
$$

where $\mathrm{d} S$ is the infinitesimal area on the sphere.

Introducing the notation $T=u_{i i}$ one can easily find that

$$
u_{i j}^{2}=\left(u_{i j}-\frac{1}{2} \delta_{i j} T\right)^{2}+\frac{1}{2} T^{2}=\frac{1}{2}\left(u_{11}-u_{22}\right)^{2}+2 u_{12}^{2}+\frac{1}{2} T^{2},
$$

whence the density of elastic energy becomes

$$
\begin{aligned}
\mathcal{E}_{\mathrm{el}}= & \sigma u_{3}^{2}+\lambda u_{3} T+\mu T^{2}+\tau u_{i j}^{2}=\sigma\left(u_{3}+\frac{\lambda}{2 \sigma} T\right)^{2}+\left(u+\frac{\tau}{2}-\frac{\lambda^{2}}{4 \sigma}\right) T^{2} \\
& +\tau\left[\frac{1}{2}\left(u_{11}-u_{22}\right)^{2}+2 u_{12}^{2}\right] .
\end{aligned}
$$

Requiring the quadratic form (10) be positive definite one gets the stability conditions

$$
\left\{\begin{array}{l}
\sigma, \tau, 2 \mu+\tau>0 \\
\lambda^{2}<2 \sigma(2 \mu+\tau)
\end{array}\right.
$$

The various contributions to the elastic energy (8) have simple physical meaning. Using (10) we get for the shear modes $u_{3}=u_{11}=u_{22}=0$ the energy density $2 \tau u_{12}^{2}$, whence $\tau$ can be called the shear modulus. Similarly, for the compression (dilation) modes $u_{3}=u_{12}=0, u_{11}=u_{22}$ one gets the energy density $2(2 \mu+\tau) u_{11}^{2}$, whence $2 \mu+\tau$ can be identified as the compression modulus; the radial modulus $\sigma$ is obtained for the radial modes $u_{i j}=0$, whose energy is $\sigma u_{3}^{2}$. An additional elasticity modulus is $\lambda$, which corresponds to the pinch modes $u_{12}=0, u_{11}=u_{22}$, $u_{3}+(\lambda / 2 \sigma) T=u_{3}+(\lambda / \sigma) u_{11}=0$. The associated energy density is

$$
\left[\frac{1}{2}(2 \mu+\tau)-\frac{\lambda^{2}}{4 \sigma}\right] 4 u_{11}^{2}=2(2 \mu+\tau) u_{11}^{2}-\sigma u_{3}^{2},
$$

which justifies the denomination of pinch modulus for $\lambda$. One notices that it has not a definite sign, and from $u_{3}=-(\lambda / \sigma) u_{11}$ one may say that $\lambda>0$ corresponds to the surface that can be pinched $\left(u_{11}<0, u_{3}>0\right)$, while the rather unrealistic 
case $\lambda<0$ describes the surface that cannot be pinched $\left(u_{11}<0, u_{3}<0\right)$. The stress tensor $f_{i j}$ is given by the derivative of $(10)$ with respect to $R u_{i j}$; among its components $f_{11}$ and $f_{22}$ correspond to the surface tensions ${ }^{\dagger}$, while the radial pressure is $f_{3}=\left(\delta \mathcal{E}_{\text {el }} / R \delta u_{3}\right)$.

From the displaced position vector given by (1) and (2) one easily obtains the kinetic energy

$$
E_{\mathrm{kin}}=\frac{1}{2} \rho R^{2} \int \mathrm{d} S\left(\dot{u}_{1}^{2}+\sin ^{2} \theta \cdot \dot{u}_{2}^{2}+\dot{u}_{3}^{2}\right)
$$

where $\rho$ is the (superficial) mass density. The identification of the elastic moduli made above is very convenient for investigating the vibrations of this hollow-molecular model, although the decomposition into the eigenfrequencies cannot easily be carried out, except for the azimuthal shear waves and the radial waves. Indeed, for the shear modes $u_{3}=0, u_{11}=u_{22}$ we see that the $u_{1}$ displacement depends only on $\varphi$ (azimuthal modes) and the $u_{2}$ displacement is a function of $\theta$ only (polar modes). The Hamiltonian of the azimuthal shear waves is therefore easily obtained from (8) and (13) as

$$
H_{\mathrm{s} 1}=\rho R^{4} \int \mathrm{d} \varphi \dot{u}_{1}^{2}+\tau R^{2} \int \mathrm{d} \varphi\left(\frac{\partial u_{1}}{\partial \varphi}\right)^{2}
$$

leading to the eigenfrequencies $\left(\tau m^{2} / \rho R^{2}\right)^{1 / 2}$, where $m$ is any integer. Similarly, the radial modes described by the Hamiltonian

$$
H_{\mathrm{r}}=\frac{1}{2} \rho R^{2} \int \mathrm{d} S \dot{u}_{3}^{2}+\sigma \int \mathrm{d} S u_{3}^{2}
$$

are easily decomposed in spherical harmonics

$$
u_{3}(\theta, \varphi)=\sum_{l m} v_{l m} Y_{l m}(\theta, \varphi)
$$

and quantized by

$$
v_{l m}=(-1)^{l+m} v_{l-m}^{+}=\frac{1}{R} \sqrt{\frac{2 \pi \hbar}{M \omega_{\mathrm{r}}}}\left[a_{l m}^{+}+(-1)^{l-m} a_{l-m}\right],
$$

where $M=4 \pi \rho R^{2}$ is the mass of the sphere and

$$
\omega_{\mathrm{r}}=\frac{1}{R} \sqrt{\frac{2 \sigma}{\rho}}
$$

is the frequency (pulsation) of the radial modes. The Hamiltonian (15) acquires the harmonic-oscillator form

$$
H_{\mathbf{r}}=\sum_{l m} \hbar \omega_{\mathbf{r}}\left(a_{l m}^{+} a_{l m}+1 / 2\right)
$$

whence one can see the high degeneracy of the radial vibration states.

Finally, we note that the independent vibrations of the sphere include two transverse shear modes, two longitudinal compression modes, two transverse radial modes and two pinch modes with indefinite polarization.

tThe present results may also apply to a spherical, molecular film, in which case it is very likely that the shear modulus vanishes as a result of the (short-range) central forces. 


\section{Rotations and deformations}

Assuming that the spherical molecule rotates with the angular velocity $\omega$ about the $z$-axis and leaving aside the translations and the vibrations the kinetic energy reduces to the centrifugal energy

$$
E_{\mathrm{cf}}=\frac{1}{2} \rho \int \mathrm{d} S(\boldsymbol{\omega} \times \tilde{\boldsymbol{R}})^{2}=\frac{1}{2} \rho \omega^{2} \int \mathrm{d} S \tilde{\boldsymbol{R}}^{2} \sin ^{2} \tilde{\theta},
$$

where $\tilde{\boldsymbol{R}}$ is given by (1) and (2). The consistency with the harmonic approximation of the elastic energy (8) requires, in principle, the expansion of $E_{\mathrm{cf}}$ to the second order in the displacements,

$$
\begin{aligned}
E_{\mathrm{cf}} & =\frac{1}{2} I \omega^{2}+\frac{1}{2} \rho \omega^{2} R^{2} \int \mathrm{d} S\left(u_{1} \sin 2 \theta+2 u_{3} \sin ^{2} \theta\right. \\
& \left.+u_{1}^{2} \cos 2 \theta+u_{3}^{2} \sin ^{2} \theta+2 u_{1} u_{3} \sin 2 \theta\right),
\end{aligned}
$$

where $I=(8 \pi / 3) \rho R^{4}=(2 / 3) M R^{2}$ is the inertia moment of the spherical molecule. Since $E_{\mathrm{cf}}$ does not contain $u_{2}$ and $\varphi$, the solutions of the Lagrange equations are $u_{2}=0$ and $u_{1,3}(\theta)$. The elastic energy (8) becomes

$$
E_{\mathrm{el}}=\sigma \int \mathrm{d} S u_{3}^{2}+\lambda \int \mathrm{d} S u_{3} \frac{\partial u_{1}}{\partial \theta}+(\mu+\tau) \int \mathrm{d} S\left(\frac{\partial u_{1}}{\partial \theta}\right)^{2}
$$

and the equilibrium equations read

$$
\begin{aligned}
& \frac{1}{\sin \theta} \frac{\partial}{\partial \theta}\left\{\sin \theta\left[\lambda u_{3}+2(\mu+\tau) \frac{\partial u_{1}}{\partial \theta}\right]\right\} \\
& +\frac{1}{2} \rho \omega^{2} R^{2}\left[\left(1+2 u_{3}\right) \sin 2 \theta+2 u_{1} \cos \theta\right]=0
\end{aligned}
$$

and

$$
\rho \omega^{2} R^{2}\left[\left(1+u_{3}\right) \sin ^{2} \theta+u_{1} \sin 2 \theta\right]-2 \sigma u_{3}-\lambda \frac{\partial u_{1}}{\partial \theta}=0 .
$$

Introducing the parameter

$$
a=\frac{\rho \omega^{2} R^{2}}{2 \sigma}
$$

the latter equation (24) can be recast as

$$
\left(1-a \sin ^{2} \theta\right) u_{3}=a \sin ^{2} \theta+a \sin 2 \theta \cdot u_{1}-\frac{\lambda}{2 \sigma} \frac{\partial u_{1}}{\partial \theta},
$$

whence one can see that we can get meaningful results only for $a \ll 1$. Noticing that the parameter $a$ given by (25) can: also be written as

$$
a=\frac{M R^{2} \omega^{2}}{2 E_{0}}=\frac{3}{4} \frac{I \omega^{2}}{E_{0}}=\frac{3}{2} \frac{E_{\mathrm{rot}}}{E_{0}},
$$

where $E_{\text {rot }}=(1 / 2) I \omega^{2}$ and $E_{0}=4 \pi R^{2} \sigma$ is the "internal" elastic energy (i.e. the elastic energy stored in a uniform radial expansion or compression of maximum relative displacement $\left|u_{3}\right|=1$ ), the condition $a \ll 1$ requires the rotation energy to be much smaller than the "internal" elastic energy, in order the deformable molecule is stable when subjected to rotations. In particular, one can see that for $a>1$ the sphere may blow up during rotation at some values of $\theta$. The parameter $a$ turns out to be the natural perturbation parameter in treating the rotations and 
the deformations of a spherical molecule, as well as the radial vibrations, as we shall see in the next section.

To the first order in $a$ we get from (26)

$$
u_{3}=a \sin ^{2} \theta+a \sin 2 \theta \cdot u_{1}-\frac{\lambda}{2 \sigma}\left(1+a \sin ^{2} \theta\right) \frac{\partial u_{1}}{\partial \theta},
$$

which introduced in (28) yields $u_{1}=a f(\theta)$, where

$$
\frac{1}{\sin \theta} \frac{\partial}{\partial \theta}\left(\sin \theta \frac{\partial f}{\partial \theta}\right)=\frac{2 \sigma(3 \lambda+2 \sigma)}{\lambda^{2}-4 \sigma(\mu+\tau)} \sin \theta \cos \theta
$$

the denominator being always negative as required by the stability conditions (11). Equation (29) can easily be solved for $f$ with the boundary condition $f(\theta)=$ $-f(\pi-\theta)$, so that we get the displacements

$$
u_{1}=a \beta(\sin 2 \theta-2 \theta+\pi)
$$

and

$$
u_{3}=a \alpha \sin ^{2} 0
$$

where

$$
\left\{\begin{array}{l}
\alpha=2 \sigma[2(\mu+\tau)+\lambda / 3] /\left[4 \sigma(\mu+\tau)-\lambda^{2}\right], \\
\beta=\sigma(\lambda / 2+\sigma / 3) /\left[4 \sigma(\mu+\tau)-\lambda^{2}\right] .
\end{array}\right.
$$

We remark that since $E_{\text {rot }} \sim a E_{0}$ only the linear approximation in displacements is relevant for the centrifugal energy $(21)^{\ddagger}$.

Having obtained the deformations $u_{1}$ and $u_{3}$ as given by (30) and (31), respectively, we are now able to specify the shape of the deformed molecule: during rotation the spherical molecule flattens, acquiring the shape of a symmetric top (with the principal axes of inertia set up by the rotation), the polar radius being given by

$$
\tilde{R}(\theta+\delta \theta) \approx \tilde{R}(\theta)=R\left[1+u_{3}(\theta)\right],
$$

the variation of the polar angle being $\delta \theta \approx u_{1}(\theta)$. We obtain straightforwardly the area of the deformed molecule

$$
\tilde{S}=S+R^{2} \int \mathrm{d} \theta \mathrm{d} \varphi\left(u_{1} \cos \theta+2 u_{3} \sin \theta\right)=S\left[1+\frac{4}{3} a(\alpha+2 \beta)\right],
$$

where $S=4 \pi R^{2}$, and the volume enclosed by the deformed molecule

$$
\tilde{V}=\frac{1}{3} R^{3} \int \mathrm{d} \theta \mathrm{d} \varphi\left(\sin \theta+u_{1} \cos \theta+5 u_{3} \sin \theta\right)=V\left[1+\frac{2}{3} a(5 \alpha+4 \beta)\right],
$$

where $V=(4 \pi / 3) R^{3}$. The tensor of inertia can also be computed easily, with the results

$$
\begin{aligned}
& I_{x x}=I_{y y}=I\left[1+\frac{2}{5} a\left(3 \alpha-\frac{8}{3} \beta\right)\right], \\
& I_{z z}=I\left[1+\frac{8}{5} a\left(\alpha+\frac{4}{3} \beta\right)\right]
\end{aligned}
$$

and $I_{x y}=I_{y z}=I_{z x}=0$.

\footnotetext{
$\ddagger$ As well as for the angular momentum.
} 
The centrifugal energy is given by $(21)$ as $E_{\mathrm{cf}}=(1 / 2) I_{z z} \omega^{2}$, while the displacements $u_{1}$ and $u_{3}$ yield from (22) the elastic energy

$$
E_{\mathrm{el}}=\frac{2}{5} a I \omega^{2}\left(\alpha^{2}-4 \frac{\lambda}{\sigma} \alpha \beta+16 \frac{\mu+\tau}{\sigma} \beta^{2}\right) .
$$

After somewhat lengthy calculation (38) becomes

$$
E_{\mathrm{el}}=\frac{2}{5} \alpha I \omega^{2}\left(\alpha+\frac{4}{3} \beta\right)
$$

so that we get the lotal energy stored by the deformed rotating molecule as

$$
E=E_{\mathrm{cf}}+E_{\mathrm{el}}=\frac{1}{2} I \omega^{2}\left[1+\frac{12}{5} a\left(\alpha+\frac{4}{3} \beta\right)\right] .
$$

The quantization of the rotation energy requires the modulus $K_{z}=\omega I_{z z}$ of the angular momentum $\boldsymbol{K}$. From (27) and (37) we get

$$
\boldsymbol{K}^{2}=I^{2} \omega^{2}\left[1+\frac{12}{5} \frac{I \omega^{2}}{E_{0}}\left(\alpha+\frac{4}{3} \beta\right)\right]
$$

whence one can see that during rotation the deformable molecule diminishes the angular velocity in order to conserve the angular momentum. Eliminating $\omega$ betwecn (40) and (41) we get the energy

$$
E=\frac{\boldsymbol{K}^{2}}{2 I}\left[1-\frac{4}{5}\left(\alpha+\frac{4}{3} \beta\right) \frac{\boldsymbol{K}^{2}}{E_{0} I}\right]
$$

where $\boldsymbol{K}^{2}=\hbar^{2} k(k+1), k$ - any positive integer. The deformed rotational states have therefore the energies

$$
E_{k}=\frac{\hbar^{2}}{2 I} k(k+1)\left[1-\frac{4}{5}\left(\alpha+\frac{4}{3} \beta\right) \frac{\hbar^{2}}{E_{0} I} k(k+1)\right],
$$

where $k=0,1,2, \ldots$ and $\alpha, \beta$ are given by (32); the $k$-state has also a $2 k+1$ orientational degeneracy.

The stability condition $a \ll 1$ reads now

$$
\frac{\hbar^{2}}{E_{0} I} k(k+1) \ll 1
$$

which implies $\hbar^{2} / E_{0} I \ll 1$, or

$$
h \omega_{\text {rot }}=h^{2} / I \ll E_{0},
$$

i.e. the rotation quanta are much smaller than the "internal" energy. Usually the rotation quanta are very small. For $\mathrm{C}_{60}$ with $R=3.5 \AA$ we get $\hbar \omega_{\text {rot }} \approx 10^{-2} \mathrm{~K}$, so that at any finite temperature $E_{\text {rot }}=\hbar \omega_{\text {rot }} \overline{k(k+1)} \sim T \ll E_{0}$. 


\section{Radial vibrations}

It is reasonable to assume as a first approximation that for a hollow, spherical molecule the surface elasticity moduli $\mu$ and $\tau$ are much larger than the radial elastic modulus $\sigma$, in which case one may leave aside the tangential motion, the molecule vibrations being described solely by the radial displacement $u_{3}=u$. The position is then given by $\tilde{R}=\boldsymbol{R}(1+u)$ and the velocity

$$
\boldsymbol{V}=\boldsymbol{\omega} \times \boldsymbol{R}(1+u)+\boldsymbol{R} \dot{u}
$$

leads to the kinetic energy

$$
\begin{aligned}
E_{\mathrm{kin}} & =\frac{1}{2} \rho \int \mathrm{d} S v^{2} \\
& =\frac{1}{2} I \omega^{2}+\frac{1}{2} \rho \omega^{2} R^{2} \int \mathrm{d} S\left(2 u+u^{2}\right) \sin ^{2} \theta+\frac{1}{2} \rho R^{2} \int \mathrm{d} S \dot{u}^{2},
\end{aligned}
$$

where one notes the absence of the Coriolis coupling. However, the second term in the right hand side of (47) is the centrifugal coupling between rotations and vibrations, including both linear and quadratic contributions in $u$. Adding the elastic energy

$$
E_{\mathrm{el}}=\sigma \int \mathrm{d} S u^{2}
$$

one obtains the Hamiltonian of the rotating molecule with radial vibrations as

$$
H=\frac{1}{2} \rho R^{2} \int \mathrm{d} S\left[\frac{2}{3} \omega^{2}+\dot{u}^{2}+\omega_{\mathbf{r}}^{2} u^{2}+\omega^{2}\left(2 u+u^{2}\right) \sin ^{2} \theta\right],
$$

where $\omega_{\mathrm{r}}=\left(2 \sigma / \rho R^{2}\right)^{1 / 2}$ is the radial vibration frequency given by (18).

Noticing that the perturbation parameter $a$ given by (25) is the square ratio of the rotation to the radial vibration pulsations, $a=\omega^{2} / \omega_{\mathbf{r}}^{2}$, Eq. (49) can also be written as

$$
H=\frac{1}{2} \rho \dot{R}^{2} \int \mathrm{d} S\left[\frac{2}{3} a \omega_{\mathrm{r}}^{2}+\dot{u}^{2}+\omega_{\mathrm{r}}^{2} u^{2}+a \omega_{\mathrm{r}}^{2}\left(2 u+u^{2}\right) \sin ^{2} \theta\right],
$$

whence one can see that while the rotation energy may be comparable to the vibration energy, the centrifugal coupling generated by rotations is a higher-order correction which lies beyond the harmonic approximation. Indeed, by substituting $u=-u_{0}+v$ in (50), where $u_{0}=a \sin ^{2} \theta$ is the static radial deformation given by (31) for $\alpha=1(\mu, \tau \gg \sigma)$, the leading contributions to (50) are

$$
H=\frac{1}{2} \rho R^{2} \int \mathrm{d} S\left(\frac{2}{3} a \omega_{\mathrm{r}}^{2}+\dot{v}^{2}+\omega_{\mathrm{r}}^{2} v^{2}\right)
$$

i.e. $H=(1 / 2) I \omega^{2}+H_{\mathrm{r}}$, where $H_{\mathrm{r}}$ is the Hamiltonian (15) of the radial vibrations. It is worth noting the general structure of $H$ given by (50) and (51) as $E_{0} \cdot\left(a, v^{2}, \ldots\right)$, where $E_{0}$ is the "internal" elastic energy. We remark in this context that the Coriolis coupling, if it were present, would bring (via the conservation of the angular momentum) a main contribution of the order $I \omega v \dot{v}=I \omega \omega_{\mathrm{r}} v^{2}=$ $\left(\omega / \omega_{\mathrm{r}}\right) E_{\text {vib }}$ to $(50)$, which amounts to an energy correction $\sim E_{0} a \sqrt{a}$ §.

\footnotetext{
${ }^{\S} \mathrm{A}$ similar estimation is obtained for the Coriolis coupling of the tangential vibrations, after the
} 
It is a straightforward matter now to see that, within the present approximation, the angular momentum reduces to that of the rigid molecule ${ }^{\text {II }}$ so that, making use of (19) and introducing the notation $n=\sum_{l m} a_{l m}^{+} a_{l m}$, we can write down the rotation-vibration energies as

$$
E_{n k}=\frac{\hbar^{2}}{2 I} k(k+1)+\hbar \omega_{\mathbf{r}}(n+N / 2),
$$

where $n$ is any positive integer. The degeneration of the vibration $n$-state is given by $(N+n-1) ! / n !(N-1)$ ! (i.e. the number of ways in which one can cast $n$ balls in $N$ boxes), where $N=\sum_{l m} l$ is the number of the degrees of freedom corresponding to the radial vibrations. Denoting by $N_{\mathrm{a}}$ the number of atoms in the molecule we get $N=N_{\mathrm{a}}-6$ by subtracting from $3 N_{\mathrm{a}}$ the degrees of freedom corresponding to translations (3), rotations (3) and those associated to the (frozen) tangential vibrations $\left(2 N_{\mathrm{a}}\right)$.

Having in view the general structure of $H$ given by (50) and (51) one can see that the harmonic-approximation condition $\overline{v^{2}} \ll 1$ requires $E_{\text {vib }}=$ $E_{0} \overline{v^{2}} \ll E_{0}$, i.e. the stability of the vibrating molecule (entirely similar with $E_{\text {rot }}=$ $(2 / 3) E_{0} a \ll E_{0}$ given by (27)). On the other hand, by using (17) we get

$$
\overline{v^{2}}=\frac{2 \pi \hbar}{M R^{2} \omega_{\mathrm{r}}} n \ll 1,
$$

which implies $\hbar / I \omega_{\mathrm{r}} \ll 1$, or $\omega_{\text {rot }} \ll \omega_{\mathrm{r}}$, a condition already contained in $a \ll 1$ $\left(\omega \ll \omega_{\mathrm{r}}\right)$. From $(18)$ one also obtains $\hbar \omega_{\mathrm{r}}=\left[(4 / 3) E_{0} \hbar \omega_{\mathrm{rot}}\right]^{1 / 2} \ll E_{0}$, so that we get

$$
\hbar \omega_{\text {rot }} \ll \hbar \omega_{\mathrm{r}} \ll E_{0}
$$

inequalities implied by $a \ll 1$. One can say that the condition $a \ll 1$ and the harmonic-approximation condition $\overline{v^{2}} \ll 1$ express the stability of the rotating and vibrating molecule. The latter condition implies also temperatures much lower than the "internal" energy, $T \ll E_{0}$.

Finally, it is worth remarking that

$$
\left\{\begin{array}{l}
a \sim \frac{\hbar^{2}}{E_{0} I} k(k+1) \sim \frac{\hbar \omega_{\text {rod }}}{E_{0}} k(k+1) \ll 1, \\
\overline{v^{2}} \sim \frac{\hbar \omega_{\text {orl }}}{\hbar \omega_{\mathrm{r}}} n \sim \sqrt{\frac{\hbar \omega_{\text {rot }}}{E_{0}}} n \ll 1,
\end{array}\right.
$$

which show that, although the rotations may involve energies comparable to the vibration energies, they always produce deformations much smaller than vibrations. This is another way of seeing why the rotation-vibration coupling generated

rotations have been separated from vibrations by $\int \mathrm{d} S \boldsymbol{R} \times \tilde{\boldsymbol{R}}=0$. The translation motion (of the center of mass of the undeformed, non-vibrating molecule) can also be included in this analysis. The translation quanta are $\hbar \omega_{t}=\hbar^{2} / M V^{2 / 3}$, where $V$ is the volume wherein the molecule moves, and one can see that we have the hierarchy $\omega_{1} \ll \omega_{r o t} \ll \omega_{r}$ (the latter inequality from $a \ll 1)$. However, the translation energy $E_{t}=M v_{0}^{2} / 2$ may take any value, in contrast with the rotation and vibration energies that are limited by $E_{0}$. While the latter conditions express the stability of the deformable molecule during rotations and vibrations, there is no such condition for translations since these are not coupled either to rotations or vibrations. In the case of the radial motion this center-of-mass decoupling reads $\int \mathrm{d} S \boldsymbol{R} u=0$.

T The deformation energy that corrects the rotation spectrum in (43) is of the order $\sim E_{0} a^{2}$, and therefore beyond the harmonic approximation. 
via deformations (as the present centrifugal coupling) is beyond the harmonic approximation.

\section{Concluding remarks}

The rotation and vibration spectra of a deformable, hollow, spherical molecule have been discussed in this paper within a continuum elasticity model which may be relevant for the $\mathrm{C}_{60}$ fullerene molecule. In particular, the discussion has been restricted to the radial vibrations, whose lowest frequency is accurately described by the continuum model. As usually, the translational degrees of freedom have been separated from the rotation and vibration ones, while the Coriolis coupling is absent for the radial vibrations. It has been shown that the remaining centrifugal coupling between rotations and vibrations produces a (relative) deformation $a=(3 / 2) E_{\text {rot }} / E_{0} \ll 1$ which is comparable with the mean square vibration $\overline{v^{2}}=E_{\mathrm{vib}} / E_{0} \ll 1$ but which implies a deformation energy $\sim E_{0} a^{2}$ much smaller than the rotation energy and the vibration energy in the harmonic approximation. It follows that if the molecular deformations have to be included (as corrections, for example, to the thermal properties corresponding to rotations and vibrations) higher-order contributions have also to be added then to the lowest-order, harmonic-approximation energy.

Finally, we note that the radial vibrations discussed within the present continuum model correspond to the "breathing" mode ( $A_{\mathrm{g}}$, Raman active) whose the discrete-structure models $[8,9]$ of $\mathrm{C}_{60}$ assign the frequency $\omega_{\mathrm{r}} \approx 60 \mathrm{meVll}$. This would correspond, according to (18) and (27), to $\sigma \approx 10^{5} \mathrm{dyn} / \mathrm{cm}$ and $E_{0} \approx 10^{3} \mathrm{eV}$; at room temperature it implies an extremely small deformation parameter $a \sim T / E_{0} \approx 10^{-4}$, which indicates a practically negligible deformation of the $\mathrm{C}_{60}$ molecule during rotation and vibrations.

\section{References}

[1] II.W. Kroto, J.R. IIeath, S.C. O'Brien, R.F. Curl, R.E. Smalley, Nature 318, 162 (1985).

[2] W. Krätschmer, L.D. Lamb, K. Fostiropoulos, D.R. IIuffman, Nature 347, 354 (1990).

[3] G.N. Murthy, A. Auerbach, Phys. Rev. B 46, 331 (1992).

[4] N. Troullicr, J.L. Martins, Phys. Rev. B 46, 1754 (1992).

[5] S. Satpatliy, V.P. Antropov, O.K. Andersen, O. Jepsen, O. Gunnarsson, A.I. Lichtenstein, Phys. Rev. B 46, 1773 (1992).

[6] Ph. Lambin, A.A. Lucas, J.P. Vigneron, Phys. Rev. B 46, 1794 (1992).

[7] M.R. Pederson, A.A. Quong, Phys. Rev. B 46, 13584 (1992).

[8] R.E. Stanton, M.D. Newton, J. Phys. Chem. 92, 2141 (1988).

[9] D.E. Weeks, W.G. Hartcr, J. Chem. Phys. 90, 4744 (1989).

II The degeneracy of the radial modes $\omega_{\mathbf{r}}$ is partly lifted by the interaction with the tangential vibrations $(\lambda$-coupling in (8)). 
[10] R.L. Cappelletti, J.R.D. Copley, W.A. Kamitakahara, F. Li, J.S. Lannin, D. Ramage, Phys. Rev. Lell. 66, 3261 (1991).

[11] G. Gensterblum, J.J. Pireaux, P.A. Thiry, R. Candano, J.P. Vigneron, Ph. Lambin, A.A. Lucas, W. Krätschmer, Phys. Rev. Lett. 67, 2171 (1991).

[12] I.V. Hertel, H. Steger, J. deVries, D. Weisser, C. Menzel, B. Kamke, W. Kamke, Phys. Rev. Lett. 68, 784 (1992).

[13] D.S. Bethune, G. Meijer, W.C. Tang, H.J. Rosen, W.G. Golden, H. Seki, C.A. Brown, M.S. deVries, Chem. Phys. Lett. 179, 181 (1991).

[14] W.P. Beyermann, M.F. Ilundley, J.D. Thompson, F.N. Diederich, G. Grüner, Phys. Rev. Lett. 68, 2046 (1992).

[15] L. Landau, E. Lifshitz, Théorie de l'Elasticité, Mir, Moskva 1967. 